\title{
Kinins and cytokines in COVID-19: a comprehensive pathophysiological approach
}

Frank L. van de Veerdonk ${ }^{1 *}$, Mihai G. Netea ${ }^{1,2}$, Marcel van Deuren ${ }^{1}$, Jos W.M. van der Meer $^{1}$, Quirijn de Mast ${ }^{1}$, Roger J. Brüggemann ${ }^{3}$, Hans van der Hoeven ${ }^{4}$

${ }^{1}$ Departments of Internal Medicine, ${ }^{3}$ Pharmacology, ${ }^{4}$ Intensive Care, Radboudumc Center for Infectious Diseases (RCI), Radboudumc, Nijmegen, The Netherlands.

${ }^{2}$ Department for Genomics \& Immunoregulation, Life and Medical Sciences Institute (LIMES), University of Bonn, Germany

Corresponding author: Frank L. van de Veerdonk, MD. PhD

(Frank.vandeveerdonk@radboudumc.nl) 


\section{Abstract}

Most striking observations in COVID-19 patients are the hints on pulmonary edema (also seen on CT scans as ground glass opacities), dry cough, fluid restrictions to prevent more severe hypoxia, the huge PEEP that is needed while lungs are compliant, and the fact that antiinflammatory therapies are not powerful enough to counter the severity of the disease. We propose that the severity of the disease and many deaths are due to a local vascular problem due to activation of B1 receptors on endothelial cells in the lungs. SARS-CoV-2 enters the cell via ACE2, a cell membrane bound molecule with enzymatic activity that next to its role in RAS is needed to inactivate des-Arg ${ }^{9}$ bradykinin, the potent ligand of the bradykinin receptor type 1 (B1). In contrast to bradykinin receptor 2 (B2), the B1 receptor on endothelial cells is upregulated by proinflammatory cytokines. Without ACE2 acting as a guardian to inactivate the ligands of B1, the lung environment is prone for local vascular leakage leading to angioedema. Angioedema is likely a feature already early in disease, and might explain the typical CT scans and the feeling of people that they drown. In some patients, this is followed by a clinical worsening of disease around day 9 due to the formation antibodies directed against the spike (S)-antigen of the corona-virus that binds to ACE2 that could contribute to disease by enhancement of local immune cell influx and proinflammatory cytokines leading to damage. In parallel, inflammation induces more B1 expression, and possibly via antibody-dependent enhancement of viral infection leading to continued ACE2 dysfunction in the lung because of persistence of the virus. In this viewpoint we propose that a bradykinin-dependent local lung angioedema via $\mathrm{B} 1$ and $\mathrm{B} 2$ receptors is an important feature of COVID-19, resulting in a very high number of ICU admissions. We propose that blocking the B1 and B2 receptors might have an ameliorating effect on disease caused by COVID-19. This kinin-dependent pulmonary edema is resistant to corticosteroids or adrenaline and should be targeted as long as the virus is 
present. In addition, this pathway might indirectly be responsive to anti-inflammatory agents or neutralizing strategies for the anti-S-antibody induced effects, but by itself is likely to be insufficient to reverse all the pulmonary edema. Moreover, we provide a suggestion of how to ventilate in the ICU in the context of this hypothesis.

\section{Introduction}

COVID-19 infects mainly elderly and people with cardiovascular risk, such as hypertension

1. The clinical spectrum and imaging are so specific that MDs recognize this disease in an instant especially now that it is widespread. Every clinician recognizes that the virus does not cause disease similar to influenza, which carries the risk that designing targeted therapies based on the pathogenesis of influenza might fail in COVID-19. Research on Sars-CoV pathogenesis which might be very similar to Sars-CoV-2 pathogenesis has focused the discussion on ACE inhibitors, recombinant ACE2, and ARBs and how they could fit in the pathogenesis of COVID-19, since these pathways were extensively studied in SARS ${ }^{2,3}$. For recombinant ACE2 this would be straight forward, it would at least be an attempt to bind and try to scavenge the virus ${ }^{3}$. However, for ACE inhibitors and ARBs it is a much more complicated story. Since most of the attention was focused on the RAS system and its interaction with modulating the vascular system and inflammation, the other major role of ACE and ACE2 for the regulation of the kinin-kallikrein system was lacking attention ${ }^{4,5}$. Moreover, the notable clinical deterioration seems to be associated with increased inflammatory status, especially around day 9, which is associated with an increased inflammatory status. Here, we describe the clinical observations that brought the clues for 
explaining the potential pathophysiological mechanisms, and offer a rationale for targeted treatment at different stages of COVID-19.

\section{Clinical observations}

When patients are admitted with symptomatic COVID-19 infection fever, dry cough, and dyspnea are most commonly observed. Importantly, we observed that dyspnea and tachypnea can differ from hour to hour and a feeling of drowning is described with sometimes sudden recovery by patients. CT scans reveals unilateral or bilateral ground-glass opacities, that might progress to more clear consolidations throughout the disease. Fluid restriction improves oxygenation and ameliorates the feeling of dyspnea. Notably, plasma concentrations of D-dimers at this stage are increased without evidence of thromboembolic events.

There is a phase during clinical admission where many patients are getting better, but some will worsen especially around day nine, although this can also occur much earlier. This worsening seems to be accompanied specifically with further increases in IL-6, CRP, ferritin, without elevated procalcitonin, indicative of a progressive inflammatory status, which is a clear different pattern of the first stage of the disease. In the ICU there are several striking observations. In contrast to patients with common forms of ARDS, most patients with severe COVID-19 show an only slightly decreased pulmonary compliance. Driving pressure is usually low. Using high PEEP may therefore substantially increase functional residual capacity resulting in hyperinflation, high strain and considerable hypercapnia through an increase in dead space ventilation, but is needed to prevent hypoxia. Furthermore, PEEP shows poor recruitability in most patients. Hereby mechanical ventilation may further contribute to lung damage. Pulmonary hypertension is not an important clinical component. 


\section{Bradykinin-induced local pulmonary angioedema}

We propose it all starts with ACE2 and its role in the kallikrein-kinin system, which to date has not investigated in the pathogenesis of SARS or COVID-19. The kinin-kallikrein system is a zymogen system that after activation leads to the release of the nona-petide bradykin that after binding to the B2-receptor on endothelial cells leads to capillary leakage and thus angioedema. The prototype diseases of local peripheral transient increased bradykinin release are hereditary or acquired angio-edema ${ }^{5}$. The clinical picture of COVID-19 is in line with a single-organ failure of the lung that is due to edema at the site of inflammation. Moreover, the presence of an elevated D-dimer without thrombosis or microangiopathy is in line with the high D-dimers in angioedema. This most likely reflects the leakage of plasma substances involved in the coagulation cascade leading to fibrin and due to kallikrein activity is processed into D-dimer and leaks back into the circulation, reflecting subendothelial activation and kallikrein activity. The ACE2 and its role in the RAS system has been suggested to play a role for more than 10 years in the pulmonary edema due to ARDS and SARS ${ }^{6}$. Pulmonary edema by ACE2 dysfunction was speculated to be due to increased hydrostatic pressure as a result of vasoconstriction of the pulmonary vasculature due to high angiotensin II (a vasoconstrictor) ${ }^{6}$. However, further experiments showed no difference in hydrostatic pressure and made the explanation of high angiotensin II with vasocontriction as a cause of pulmonary edema unlikely ${ }^{6,7}$. Increased bradykinin however could explain this observation without increased hydrostatic pressure. Notably, the RAS system controls vasoconstriction and vasodilatation, and the bradykinin system controls permeability and vasodilatation, whereas ACE2 regulates both. 
Bradykinin (BK) is a linear nonapeptide that is formed by the proteolytic activity of kallikrein on kininogens ${ }^{8}$. Kallikreins are serine proteases and can be divided in plasma kallikrein and tissue kallikreins (Figure 1). The plasma and tissue kallikreins release the vasoactive peptides known as kinins (all sorts of BKs) that cause relaxation of vascular smooth muscle and increased vascular permeability ${ }^{4,9}$. Plasma kallikrein processes highmolecular-weight kininogen (HMWK produced by the liver ${ }^{8}$ ) into bradykinin, while tissue kallikrein processes low-molecular-weight kininogen (LMWK produced by the $\operatorname{liver}^{8}$ ) and results in Lys-BK (Figure 1). These are the ligands for the constitutively expressed bradykinin receptor B2 on endothelial cells ${ }^{5}$. In addition, the enzymes (carboxypeptidase $\mathrm{M}$ (CPM) and carboxypeptidase $\mathrm{N}(\mathrm{CPN})$ ) can further process BK and Lys-BK into des-Arg9BK and Lys- des-Arg9-BK respectively, which are ligands for bradykinin receptor B1, a receptor on endothelial cells that is upregulated under proinflammatory conditions ${ }^{5}$. These kinins have strong vasopermeable and vasodilatory capacity and need to be tightly controlled to prevent excessive angioedema. ACE and ACE2 both have roles in inactivating the ligands for the bradykinin receptors ${ }^{10}$. ACE mainly inactivates bradykinin which is the major ligand for $\mathrm{B} 2$ receptors. ACE inhibition has been linked to systemic acquired angioedema since it can result in excessive presence of bradykinin that activates B2 receptor ${ }^{5}$.

Interestingly, ACE2 does not inactivate bradykinin, but can inactivate des-Arg ${ }^{9}$-BK which is a potent ligand of the $\mathrm{B} 1$ receptor in the lung (Figure 1) ${ }^{11}$. In this way it can be protective against pulmonary edema especially in the setting of inflammation, which is further supported by the role of ACE2 in acute pulmonary injury ${ }^{6,11}$. ACE2 is almost undetectable in serum, but is expressed in the lung on bronchiolar exocrine cells and pneumocytes type II ${ }^{11}$. The Sars-CoV-2 Spike (S) antigen binds to ACE2 and internalizes ${ }^{12}$. Since it has been reported and suggested that the expression of ACE2 and its capacity of enzyme activity is 
decreased in SARS-CoV and inflammatory conditions ${ }^{11,6,7}$, it is tempting to speculate that Sars-CoV-2 interaction with ACE2 at the surface also downregulates ACE2 expression and function of ACE2, subsequently leading to a deficiency to inactivate the B1 ligand locally in the lung, and might in this way directly link the virus to local pulmonary angioedema.

In 2005 it was proposed that the RAS system was responsible for complications due to SarsCoV. RAS regulates vasodilatation and vasoconstriction, and it was hypothesized that increased angiotensin II as a result of ACE2 deficiency would result in pulmonary edema due to increased hydrostatic pressure since angiotensin II would cause vasoconstriction.

However, there was no effect observed on the hemodynamics of the pulmonary vasculature in ACE2 deficiency, while there was clear vascular leakage. AT1R knockout mice and AT1R blockade were protected from lung edema due to inflammation but this was not explained by a mechanism linking AT1R to vascular leakage. Bradykinin might be the missing link, since AT1R forms heterodimers with the B2 receotor and AT1R can syergise with B1 receptor in the induction of ROS in endothelial cells ${ }^{13,14}$.

We speculate that this dysregulated bradykinin pathway is present already early in COVID19 disease. Patients can worsen clinically after days of illness (especially around day 9) which is accompanied by an increase in proinflammatory status often resulting in ICU admission and with necessity of supportive mechanical ventilation. This second hit is reminiscent to observations in SARS-CoV where $80 \%$ of patients with SARS-CoV that developed acute respiratory disease coincided with antiviral IgG seroconversion ${ }^{15}$. Moreover, patients who developed the anti-S-neutralizing antibody early in disease had a higher chance of dying from the disease ${ }^{15}$. In a macaques model published a few months ago it was clear that the anti-S-neutralizing antibody lead to worsening of pulmonary disease ${ }^{16}$. 
The proposed mechanism was an induced hyperinflammation via FcG receptor ${ }^{16}$. Prior administration of anti-S-antibodies lead to an overwhelming influx of monocytes and macrophages to the lung already at day 2 of SARS-CoV infection. This abundance of monocyte/macrophages has also been reported in autopsy reports in three patients with COVID-19 from China (PMID 32172546). In this context there might also be a role for an exaggerated complement activation ${ }^{10}$. In addition, a phenomenon named antibody-dependent enhancement of viral infection might be responsible for persistent viral loads and subsequently cause a direct or indirect effect on ACE2 activity in the lung ${ }^{15}$. Important to note is that anti-inflammatory strategies might thus especially have a role at the time of seroconversion and the development of anti-S antibodies (Figure 2). This will not only result in less damage to the environment but will also protect against further inflammation-induced B1 upregulation on endothelial cells. However, it must be kept in mind that it will not have any direct effect on the pulmonary edema that is driven by bradykinin, since kallikrein activity is not affected, kinins will still be present, and B1 and B2 receptors are still expressed on endothelial cells. This pathway is not responsive to corticosteroids or adrenaline, meaning as long as the virus persists ACE2 dysfunction is present and the bradykinin pathway is active and pulmonary edema at the site of infection will persist. On the other hand, clinicians know how fast patients with bradykinin-related angioedema can recover once this pathway is blocked or the trigger is gone that one can foresee a very fast recovery of pulmonary edema and recovery of hypoxia and disease.

\section{Targeted treatment and timing of interventions}

Blocking $B 1$ and $B 2$ receptors 
In our vision, as long as the virus persists the dysregulated kinin-kallikrein pathway is playing a role in disease via the absence of optimal ACE2 function in the lung. Maybe not everybody needs B1 and B2 receptor blocking since they will recover once the viral load is resolved from the lung and there is no second inflammatory hit or significant production of anti-S-antibodies. However, when disease progresses which is accompanied by increased proinflammatory status which often results in critical illness we would argue that this timepoint has a rationale for aggressive innate anti-inflammatory strategies, however this must be done in the presence of blocking the bradykinin pathway. Several targets in the kallikrein-kinin pathway might be amendable to intervention, namely 1 . at the level of blocking tissue kallikrein activity and thus reducing the production of kinins, 2 . activating the degradation of kinins by treating with recombinant active enzymes such as ACE2, 3. at the level of B1 and B2 receptors, 4. by inhibiting the common downstream signaling of B1 and B2 receptors, and 5. by suppressing local NO which is largely responsible for the endothelal leakage.

By far the most potent and logical would be to block B1 and B2 receptor signaling. B2 receptor inhibitors exist in the clinics. Icatibant is a selective B2 receptor drug that is available in the US and Europe (Firazyr) ane is licensed for the treatment of hereditary angioedema in adults, adolescents and children over the age of 2 years. It is a synthetic decapeptide with a structure similar to bradykinin, but with 5 non-proteinogenic amino acids. [EMA assesmet report available at https://www.ema.europa.eu/en/documents/variationreport/firazyr-h-c-899-ii-0024-g-epar-assessment-report-variation_en.pdf]. The licensed dose of icatibant for hereditary angioedema is $30 \mathrm{mg}$ by subcutaneous injection as a single dose.. At current day, there is no licensed B1 receptor drug ${ }^{17}$. Several B1 receptor drugs have been tested in pre-clinical and in phase I/II trials as therapeutic target for inflammation related 
processes already since the 1970 s. None of these drugs have made it to the market. This includes drugs like the Merck compound MK-0686 ${ }^{18}$ that has been investigated in the reduction of pain, and the Sanofi compound safotibant that was discontinued in 2012 for the treatment of macular oedema and the Boehringer Ingelheim drug BI11382 (PMID:26468713). Other products identified via open target (accessible via http://www.opentarget.com) and through literature review are ELN-441958, SSR 240612, NVP-SAA164 and R-715 ${ }^{17}$. Dual inhibition of both the B1 and B2 receptor would be the way forward. But this would imply that specifically the drugs targeting B1 receptor need to be become available and that they have to exert suitable pharmacodynamic action at concentrations that are non-toxic.

\section{Anti-inflammatory strategies}

In addition, we should think about blocking innate cytokines that upregulate B1 on endothelial cells at the site of inflammation in combination with B1 and or B2 receptor blockade. IL-1 (consisting of IL-1 $\alpha$ and IL-1 $\beta$ ) and TNF are potent inducers of B1. Blockade of NF- $\kappa \mathrm{B}$ translocation, TNF- $\alpha$, or IL-1 prevented the functional and molecular up-regulation of $\mathrm{B} 1$ receptors by LPS ${ }^{19}$. Therefore, one strategy could be with anakinra, which has an excellent safety profile and would make a lot of sense since it not only blocks IL-1 $\beta$, but also IL-1 $\alpha$. IL-1 $\alpha$ is likely to be extremely elevated locally due to its release from damaged cells. Blocking TNF is an option, but has been associated with much more infectious complications. In addition, complement activation has been described and could play a role in this stage of disease, and this might be amendable to C5 blockade with eculizimab with which a randomized trial in COVID-19 is being performed (NCT04288713). Also corticosteroids are an option. Since we notice that some patients have persistent disease and at some point develop a proinflammatory profile especially a rise in CRP reflecting IL-6 
elevation), which often leads to ICU admission this might be the timepoint to initiate potent anti-inflammatory therapy. For most patients this timepoint will be identified before the need of ICU admission and thus an anti-inflammatory drug might prevent them from ICU admission. This anti-inflammatory strategy must be initiated together with B1 and B2R blockade and available antivirals. The anti-inflammatory strategies will buy time, but will not resolve the disease by themselves as long as the virus is present and or the bradykinin angioedema is not resolved. A summary of these proposed targeted treatments and timing of treatment is depicted in Figure 2. An overview of the hypothesis on which this strategy is based is illustrated by Figure 3A (normal condition), 3B (mild inflammation) and 3C (severe inflammation).

\section{Conclusions}

We are calling out for experts in the field of the kallikrein-kinin system and people involved in drug development to work together with SARS researchers who have the tools to test this hypothesis and interventions. This hypothesis explains the clinical spectrum that is so often observed and offers a rationale for treatment and more importantly timing of treatment. In addition to the bradykinin-driven pulmonary edema and cytokine-related clinical detoriation we offer a suggestion how to ventilate in this context. "Preventive" mechanical ventilation should be discouraged because the risk for ventilator induced lung injury may be higher than anticipated. After intubation, patients should be turned prone to improve oxygenation and keep PEEP at the lowest possible level. Early assisted ventilation should be stimulated unless a high respiratory drive $\left(\mathrm{P}_{0.1}<-4 \mathrm{~cm} \mathrm{H} \mathrm{H}_{2} \mathrm{O}\right.$ or a predicted $\mathrm{Pmus}>15 \mathrm{~cm} \mathrm{H} \mathrm{H}_{2}$ or a predicted transpulmonary pressure $>17 \mathrm{~cm} \mathrm{H}_{2} \mathrm{O}$ is present. In those cases, appropriate respiratory drive suppression may be attempted. Patients should be kept as dry as possible. 
1 Guan W-J, Ni Z-Y, Hu Y, et al. Clinical Characteristics of Coronavirus Disease 2019 in China. N Engl J Med 2020; : 1-13.

2 Fang L, Karakiulakis G, Roth M. Are patients with hypertension and diabetes mellitus at increased risk for COVID-19 infection? Lancet Respir 2020; 2600: 30116.

3 Batlle D, Wysocki J, Satchell K. Soluble angiotensin-converting enzyme 2: a potential approach for coronavirus infection therapy? Clin Sci (Lond) 2020; 134: 543-5.

4 Marceau F, Bawolak MT, Fortin JP, et al. Bifunctional ligands of the bradykinin B 2 and B 1 receptors: An exercise in peptide hormone plasticity. Peptides 2018; 105: 37 50.

5 Jurado-Palomo J, Caballero T. Pathophysiology of Bradykinin-Mediated Angioedema: The Role of the Complement System. A Compr Rev Urticaria Angioedema 2017. DOI: $10.5772 / 67704$.

6 Imai Y, Kuba K, Rao S, et al. Angiotensin-converting enzyme 2 protects from severe acute lung failure. Nature 2005; 436: 112-6.

7 Kuba K, Imai Y, Rao S, et al. A crucial role of angiotensin converting enzyme 2 (ACE2) in SARS coronavirus-induced lung injury. Nat Med 2005; 11: 875-9.

8 Bhoola KD, Figueroa CD, Worthy K. Bioregulation of kinins: Kallikreins, kininogens, and kininases. Pharmacol. Rev. 1992; 44: 1-80.

9 Bhoola KD, Figueroa CD, Worthy K. Bioregulation of kinins: Kallikreins, kininogens, and kininases. Pharmacol. Rev. 1992.

10 Gralinski LE, Sheahan TP, Morrison TE, et al. Complement activation contributes to severe acute respiratory syndrome coronavirus pathogenesis. MBio 2018; 9: 1-15.

11 Sodhi CP, Wohlford-Lenane C, Yamaguchi Y, et al. Attenuation of pulmonary ACE2 activity impairs inactivation of des-arg9 bradykinin/BKB1R axis and facilitates LPSinduced neutrophil infiltration. Am J Physiol - Lung Cell Mol Physiol 2018; 314: L17- 
31.

12 Walls AC, Park Y-J, Tortorici MA, Wall A, McGuire AT, Veesler D. Structure, Function, and Antigenicity of the SARS-CoV-2 Spike Glycoprotein. Cell 2020; : 1-12.

13 Quitterer U, Abdalla S. Vasopressor meets vasodepressor: The AT1-B2 receptor heterodimer. Biochem Pharmacol 2014; 88: 284-90.

14 Ceravolo GS, Montezano AC, Jordão MT, et al. An Interaction of Renin-Angiotensin and kallikrein-kinin systems contributes to vascular hypertrophy in angiotensin IIInduced Hypertension: In vivo and in vitro studies. PLoS One 2014; 9.

DOI:10.1371/journal.pone.0111117.

15 Fu Y, Cheng Y, Wu Y. Understanding SARS-CoV-2-Mediated Inflammatory Responses: From Mechanisms to Potential Therapeutic Tools. Virol Sin 2020; 12250. DOI:10.1007/s12250-020-00207-4.

16 Liu L, Wei Q, Lin Q, et al. Anti-spike IgG causes severe acute lung injury by skewing macrophage responses during acute SARS-CoV infection. JCI insight 2019; 4. DOI:10.1172/jci.insight. 123158.

17 Qadri F, Bader M. Kinin B1 receptors as a therapeutic target for inflammation. Expert Opin. Ther. Targets. 2018. DOI:10.1080/14728222.2018.1409724.

18 Kuduk SD, Di Marco CN, Chang RK, et al. Development of orally bioavailable and CNS penetrant biphenylaminocyclopropane carboxamide bradykinin B1 receptor antagonists. $J$ Med Chem 2007. DOI:10.1021/jm061094b.

19 Passos GF, Fernandes ES, Campos MM, et al. Kinin B 1 Receptor Up-Regulation after Lipopolysaccharide Administration: Role of Proinflammatory Cytokines and Neutrophil Influx . J Immunol 2004; 172: 1839-47. 
Figure 1. The kinin-kallikrein system and ACE/ACE2.

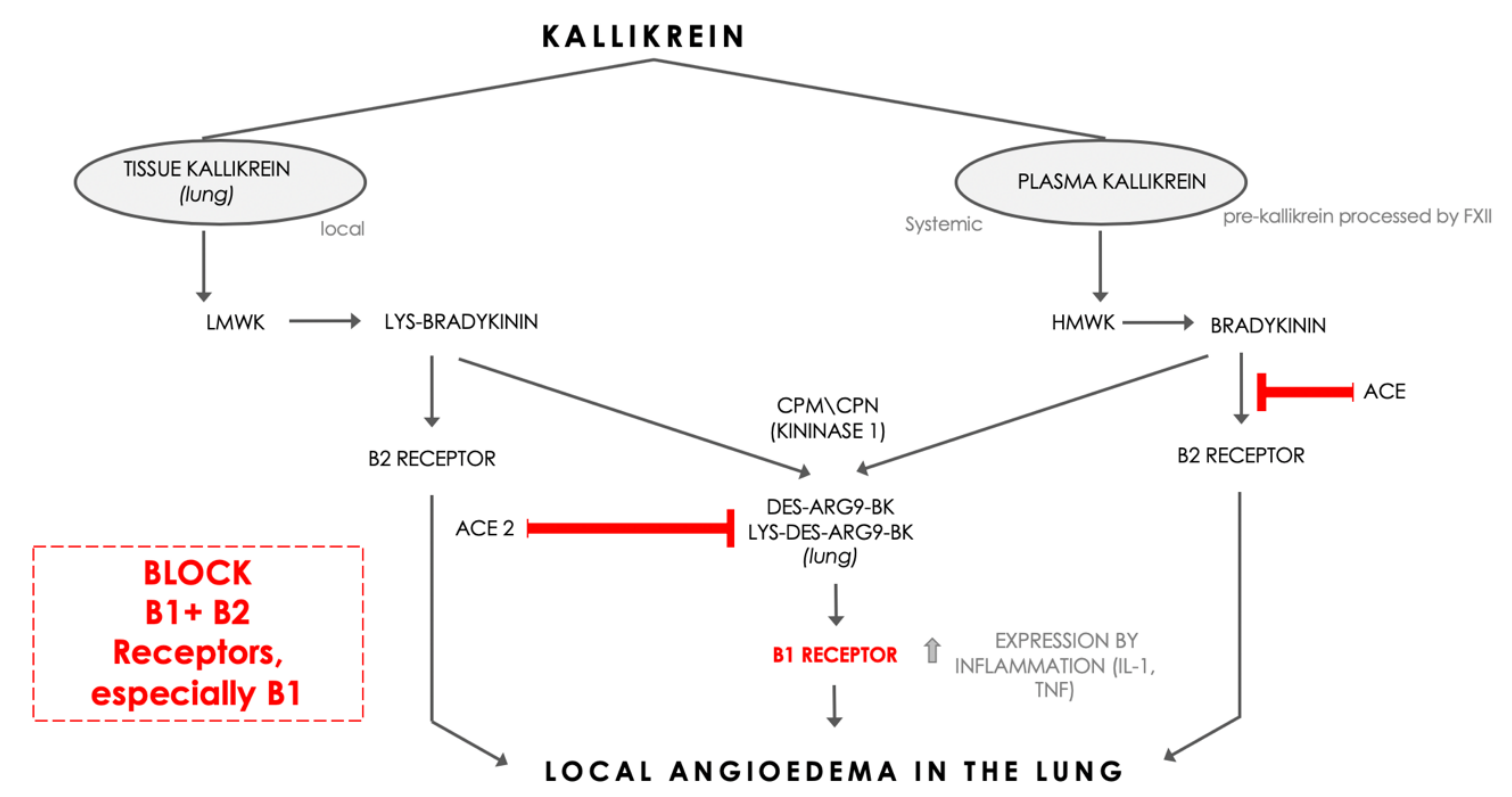

The pathways of processing of low molecular weight kininogen (LMWK) and high molecular 
weight kallikrein (HMWK) leading to Bradykinin 1 (B1) receptor agonists and Bradykinin 2 (B2) receptor agonists. $\mathrm{CPM}=$ carboxypeptidase $\mathrm{M}$; $\mathrm{CPN}=$ carboxypeptidaseN.

Figure 2. Schematic of proposed targeted treatments and timing of treatment in COVID-19.

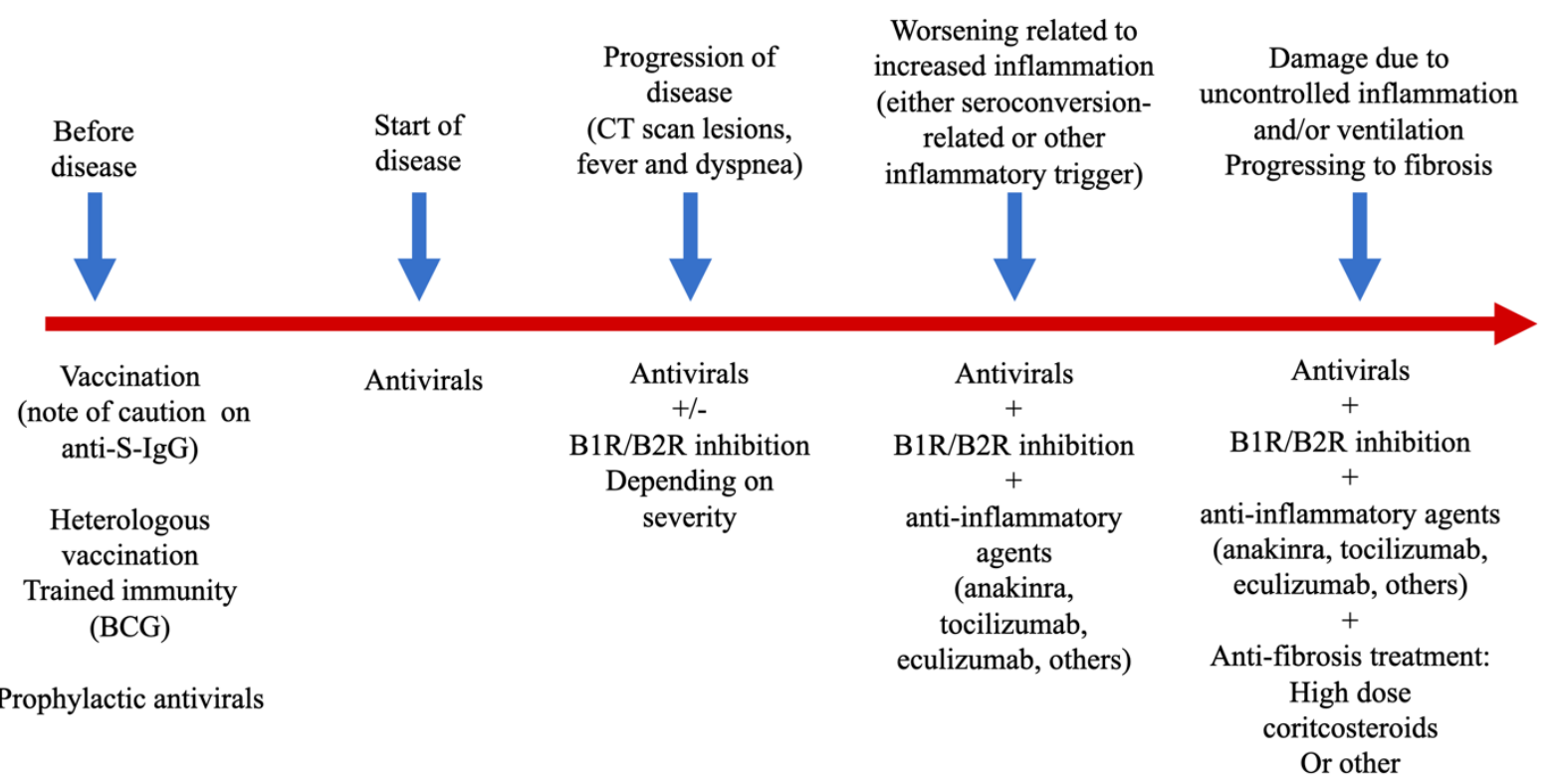


Figure 3 Illustration of hypothesis. 


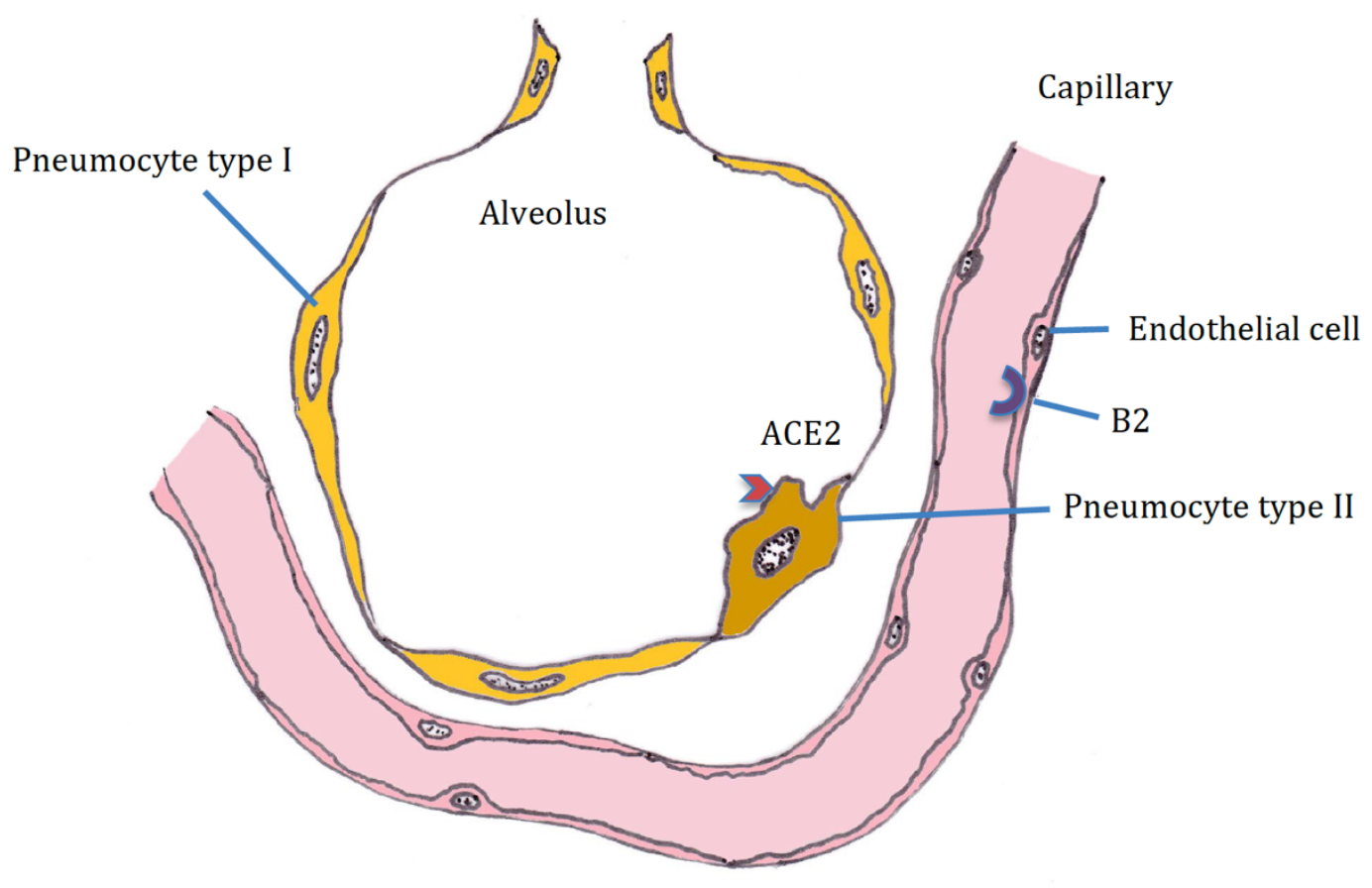

\section{A. Normal state}

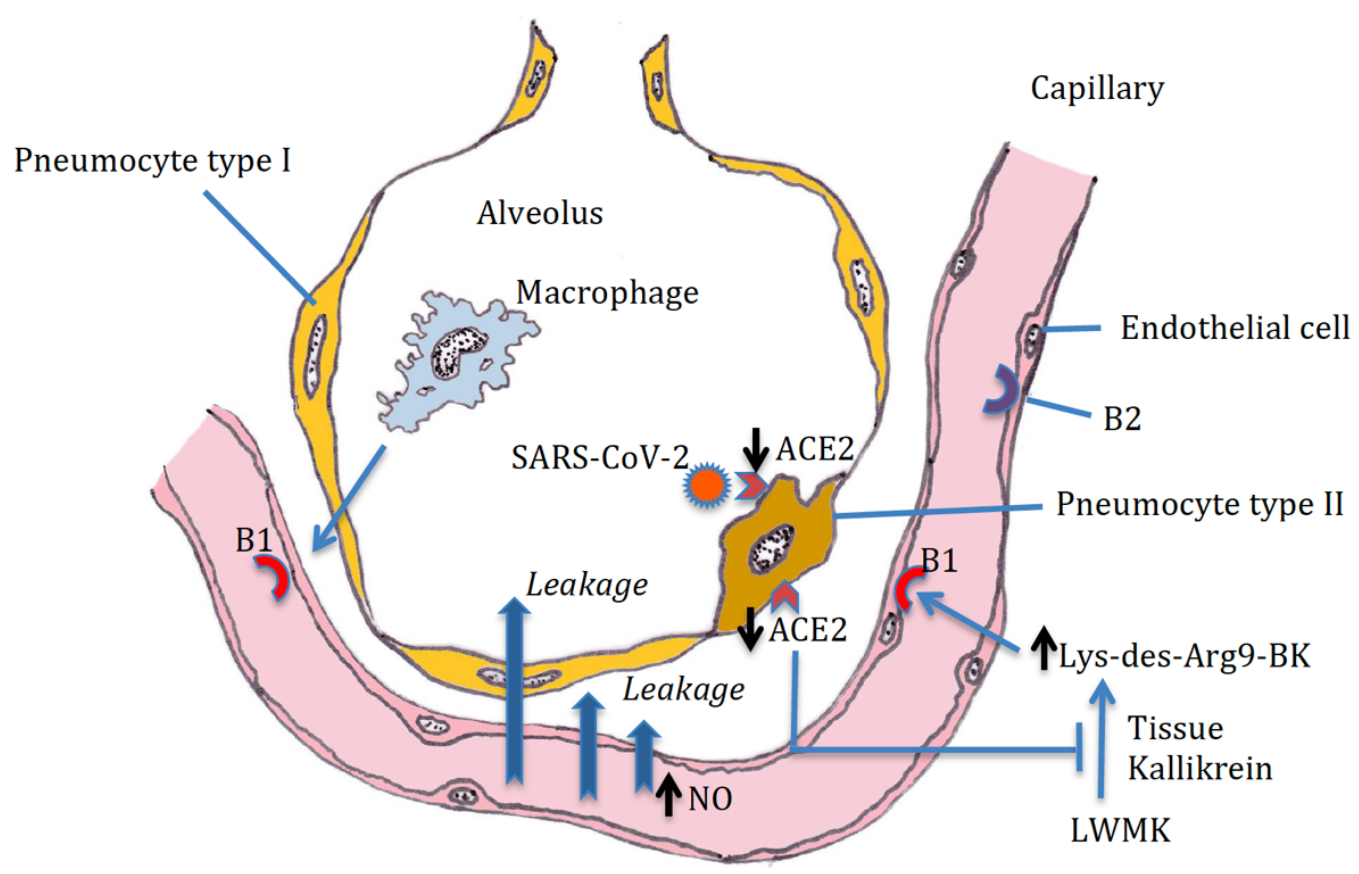

B. Mild inflammation 


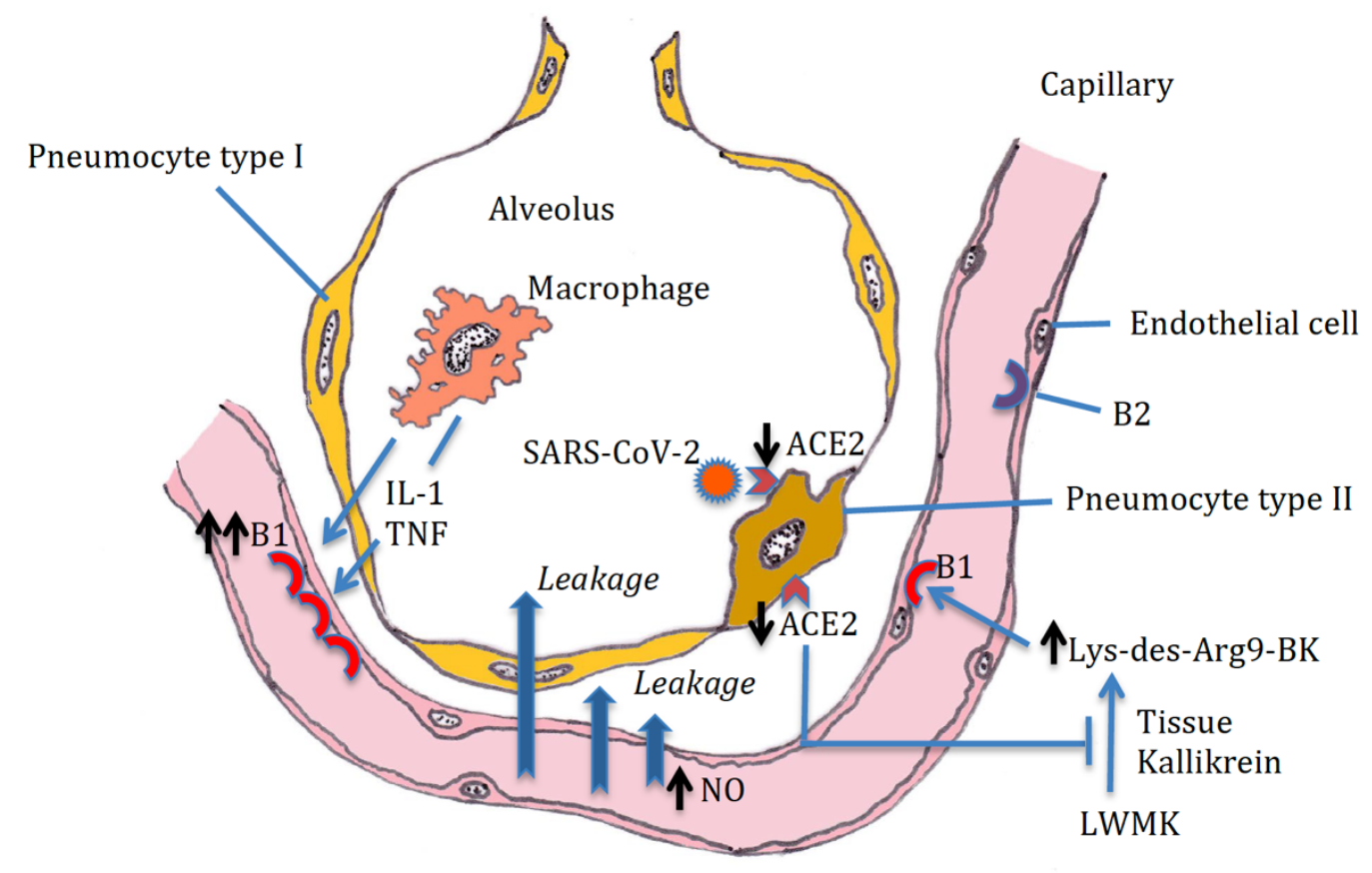

C. Hyperinflammation 